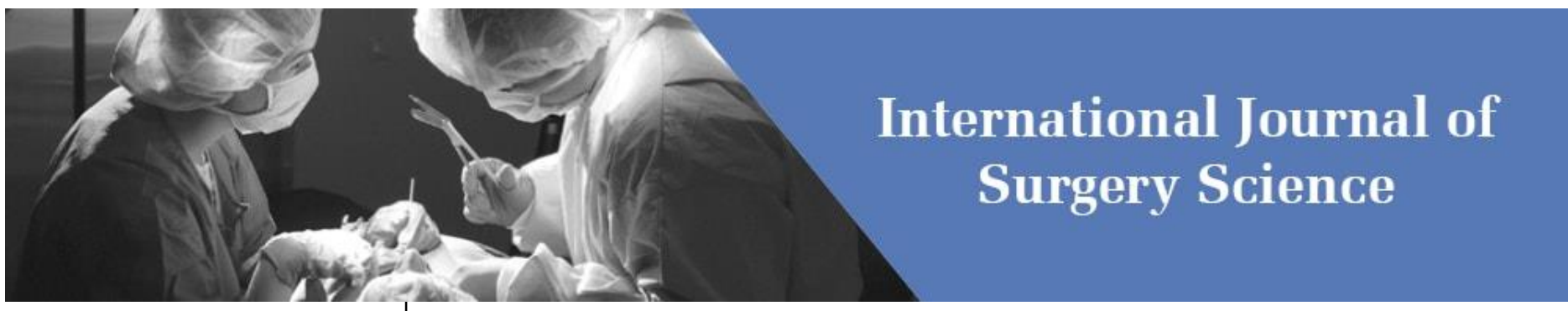

E-ISSN: 2616-3470

P-ISSN: 2616-3462

(C) Surgery Science

www.surgeryscience.com

2020; 4(3): 90-93

Received: 10-05-2020

Accepted: 13-06-2020

Dr. Ketan Vagholkar

Professor, Department of Surgery,

D.Y. Patil University School of

Medicine, Mumbai, Maharashtra, India
Corresponding Author: Dr. Ketan Vagholkar

Professor, Department of Surgery,

D.Y. Patil University School of

Medicine, Mumbai, Maharashtra,

India

\section{Pilonidal sinus}

\section{Dr. Ketan Vagholkar}

DOI: https://doi.org/10.33545/surgery.2020.v4.i3b.475

\section{Abstract}

Pilonidal sinus is disease commonly affecting young hairy individuals. The disease causes recurring infection and has a high recurrence rate. Understanding the pathophysiology is essential for developing a treatment plan. A variety of techniques have been developed for treating pilonidal sinus. These range from minimally invasive to excisional techniques. However no single procedure can be considered as the gold standard of care. Proper preparation of the area followed by optimum choice of the procedure can help in reducing both local wound complications as well as recurrences.

Keywords: Pilonidal sinus surgical treatment

\section{Introduction}

Pilonidal sinus (PS) is a descriptive term used to describe a wide spectrum of clinical presentations ranging from symptomatic hair containing sinuses to large symptomatic abscesses situated in the sacrococcygeal region ${ }^{[1,2]}$. It has a very high recurrence rate. The exact pathogenesis of the lesion still remains debatable ${ }^{[3]}$. The possible etiopathogenesis, clinical features and the various therapeutic options are discussed in this paper.

\section{Etiopathogenesis}

The disease is most commonly seen in men. (M/F 3-4:1) The disease is commonly seen in the late teens to early twenties decreasing after the age of 25 years. The average age of presentation is 21 years in males and 19 years for females ${ }^{[3,4,5]}$. It rarely occurs after the age of 45 years. Sedentary life style with obesity in a hairy male strongly predispose to the disease ${ }^{[6,7,8]}$. Few studies reveal a positive family history of pilonidal sinus ${ }^{[8,9]}$. (Table 1 )

Table 1: Risk factors

\begin{tabular}{|c|c|}
\hline Male sex & Deep natal cleft \\
\hline Hairy male & Family history \\
\hline Sitting occupation & Presence of hair in the natal cleft \\
\hline Obesity & Increased sweating \\
\hline
\end{tabular}

Various theories have been postulated to explain the pathogenesis of pilonidal sinus. Patey and Scarf hypothesized that PS was an acquired disease ${ }^{[10]}$. It is caused by penetration of hair into the subcutaneous tissues with subsequent granulomatous reaction. This explains the predilection for certain sites such as sacrococcygeal region and the finger webs of barbers and sheep breeders [11,12].

The development of PS follows a particular sequence of events.

Hair penetrates into the subcutaneous tissues through dilated hair follicles particularly during adolescence ${ }^{[13,14]}$. Upon persistent sitting or standing hair follicles break and open a pit. Debris usually collect in this pit followed by inflammatory reaction and infection. The persistent inflammatory reaction leads to the development of a sinus with a short locally extending tract. Complex, ill understood mechanisms lead to further penetration of hair into the subcutaneous tissues. The sinus extends cephalad due to mechanical forces involved in sitting or bending. Persistent inflammation due to a foreign body type of reaction can lead to the formation of local abscess. The abscess may at times rupture and drain spontaneously which serves as a portal for further invasion thereby adding to the inflammatory reaction. Persistent infection leads to recurrent abscesses and the development of a well formed sinus tract. 
The abscesses are usually formed lateral to the midline ${ }^{[15,16]}$. When they rupture releasing the pus small tracts persist which remain connected to the midline tract. These are called secondary tracts which open laterally but remain connected to the midline primary tract. Therefore understanding the exact pathology of PS is essential for developing an optimum surgical procedure ${ }^{[17,18]}$.

Microscopic study of the tract adds to the better understanding of the disease process in PS. The sinus where the hair enter is usually lined by stratified squamous epithelium with slight cornification [17]. Additional or secondary sinus tracts are frequently seen in longstanding cases. Many a time's patients present with a cyst. These cysts lack epithelial lining as seen in the sinus. Cellular infiltrate consists of PMN's, lymphocytes and plasma cells in variable proportions. Foreign body giant cells are seen in long standing and recurrent PS cases. The commonest organism grown from the sinus is staphylococcus aureus. Besides this there may be other organisms like anaerobic cocci, aerobes and many a times a mixed flora. Therefore three events underline the development of PS. The invader (hair), the force (causing hair penetration) and the vulnerability of the skin $[16,17]$

\section{Clinical features}

Pilonidal sinus can present in a variety of forms.

1. Asymptomatic cases with a just a sinus containing a tuft of hair. There may not be any inflammatory reaction in the surrounding tissues.

2. Symptomatic cases with a well formed sinus containing hair discharging seropurulent fluid.

3. A cystic swelling in the region with redness and tenderness.

4. An abscess with a fluctuant and tender swelling with surrounding induration. In a few cases the abscess will rupture discharging purulent fluid with local signs of inflammation.

5. An irregular area of redness and induration underlying a scar of previous surgery in recurrent cases.

The sinus has tendency to always head cephalad and never towards the perianal region.

Majority of patients are afebrile unless they present with an abscess. Local examination will reveal the sinus in the typical position with a tuft of hair projecting through it. (Figure 1) There will be tenderness and induration in the adjacent region. Seropurulent discharge can be expressed from the sinus opening by applying pressure on the surrounding tissues.

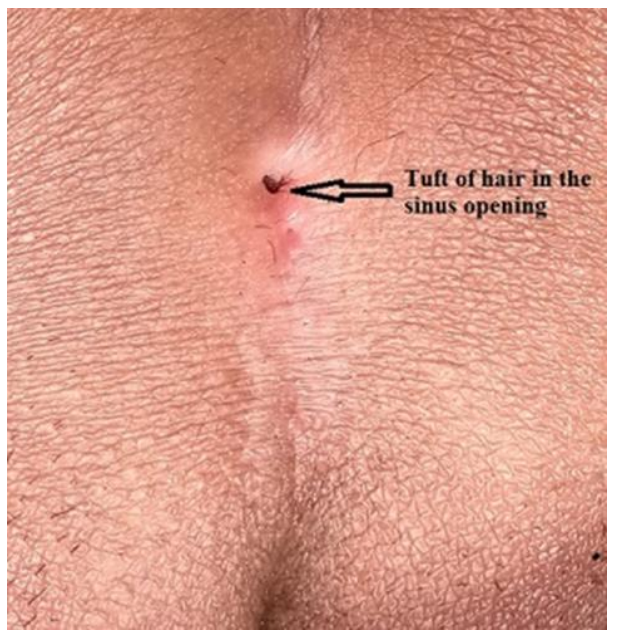

Fig 1: Pilonidal sinus with tuft of hair projecting from the sinus opening

\section{Treatment}

\section{Acute presentation}

Patients presenting as an abscess require to undergo incision and drainage. The wound needs to heal by secondary intention ${ }^{[18,19]}$. However the healing is always suboptimal leading to the formation of a well formed sinus. The sinus is always in the midline and the tuft of hair are usually seen emerging from the sinus opening. Therefore a patient presenting as an abscess will invariably require a definitive surgery. However the recurrence rate in such cases is very high.

\section{Chronic presentation}

Local inflammation and induration are common accompaniments of pilonidal sinus. The inflammatory reaction has to be reduced as much as possible in order to restrict the volume of adjacent tissue to be excised. Therefore all patients who are candidates for surgery should undergo a trial of conservative therapy wherein the local inflammation or infection is reduced. This also helps in better healing of the flaps. Excisional techniques performed in cases where local infection and induration are significantly present have a high chance of wound dehiscence as well as a recurrence. Local treatment comprises of dilute hydrogen peroxide solution irrigation of the sinus tract followed by the use of hygroscopic agents which reduce the infection and induration in the region. Analgesics and anti-inflammatory medications may be helpful if there is severe pain and discomfort. Antibiotics are indicated if there is considerable infection manifested as discharge of copious volume of foul smelling pus from the sinus tract.

Since the recurrence rate is very high there is lack of unanimity for the choice of a single procedure. A wide range of procedures have been described. Few have been termed as minimally invasive techniques, while the others are described as excisional with closure by various techniques including flaps. Irrespective of the choice of the procedure, delineation of the sinus tract which includes both the primary and secondary tracts has to be done. This is best done by instillation of methylene flue into the sinus tract. (Figure 2) Addition of a few drops of hydrogen peroxide to the methylene blue aids in ensuring complete delineation of the sinus tracts. After excision of the tract with adjacent tissue the surgeon needs to confirm that there is no residual bluish tissue. (Figure 3) This confirms complete excision of the diseased tissue before commencing closure.

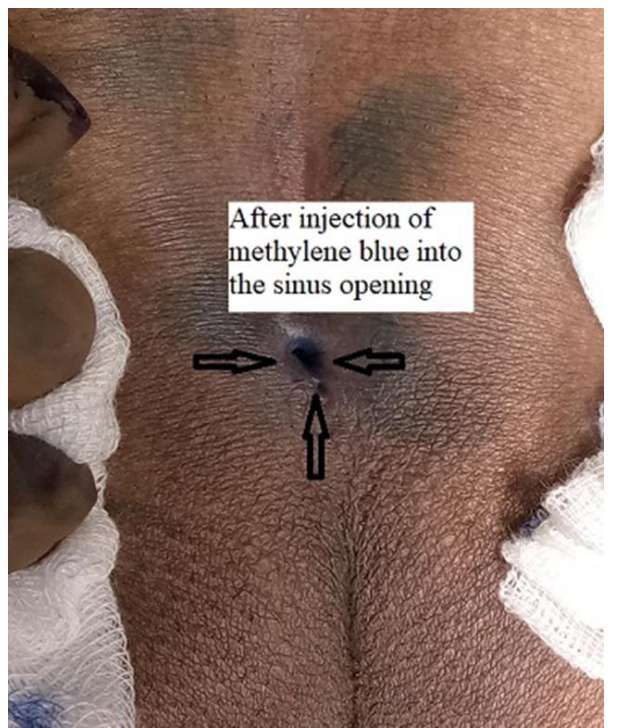

Fig 2: Methylene blue instilled into the sinus opening 


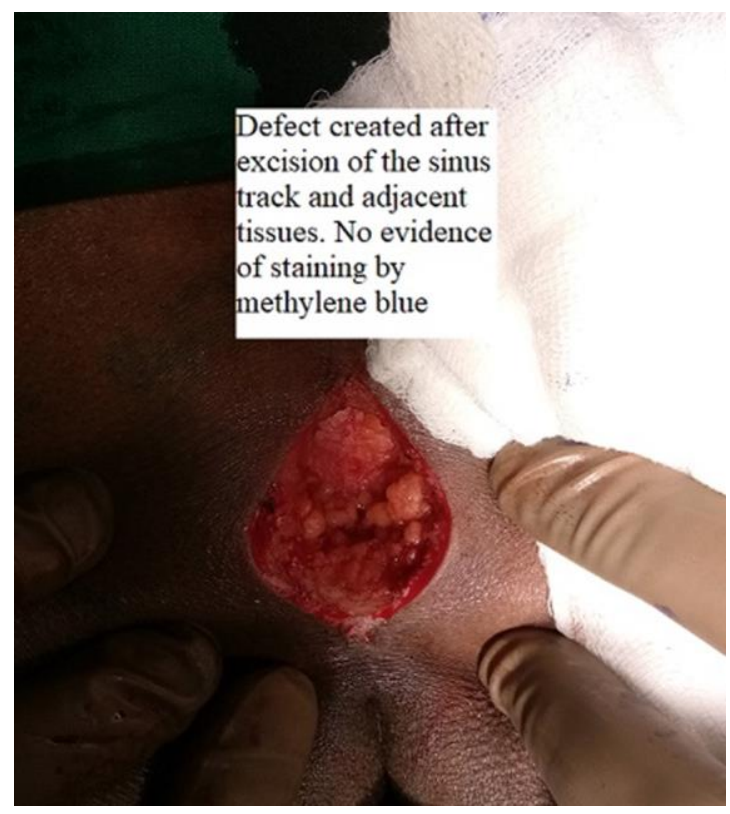

Fig 3: No evidence of residual blue stained tissues

\section{Minimally invasive techniques \\ Pit picking technique}

Sinus openings in the natal cleft are typically described as pits. In pit picking technique the midline pits are excised under local anaesthesia with a $1 \mathrm{~mm}$ rim of surrounding tissue ${ }^{[19]}$. Incisions of 1-2 cms are also made over the secondary lesions and the subcutaneous tissues are scooped and irrigated. The recurrence rate with this technique is 10 to $20 \%$. The only advantage of the procedure is that the healing time is much shorter. However this method can only be used in smaller lesions with almost no inflammatory reaction in the surrounding tissues.

\section{Sinusectomy}

In this technique the sinus tract from the primary opening to the secondary opening is excised without removing a significant portion of adjacent soft tissue ${ }^{[20]}$. The wound is left open to heal by secondary intention. The recurrence rate with this procedure is $7 \%$.

\section{Video assisted procedures}

The combination of minimally invasive techniques with laser ablation of the subcutaneous tracks has been used recently ${ }^{[21]}$. A video assisted endoscopic ablation procedure adds to the accuracy. The healing rate with this type of procedure is 80 $90 \%$. However the cost of equipment is a major limiting factor [22].

\section{Chemical cauterization}

Instillation of phenol into the sinus or fistula induces severe chemical inflammation leading to scarring ${ }^{[23]}$. This can be done under local anaesthesia. However studies in favour of this technique are inadequate to advise it as a standard of care.

\section{Excisional techniques}

Excisional techniques are of two types. Midline excisional techniques in which the suture line is in the midline while offmidline techniques wherein the suture line is lateral to the midline ${ }^{[24,25]}$.

\section{Excision with midline suture}

This involves excision of the sinus tract with midline closure of the defect. The purpose of this technique is to shorten the healing time. However the wound dehiscence and recurrence rate is quite high ${ }^{[24]}$

\section{Karyadakis procedure}

This involves asymmetrical excision of the sinus tract with closure ensuring that the suture line is lateral to the midline. The recurrence rate with this procedure is low ${ }^{[26,27,28,29]}$.

\section{Cleft lift}

This is a modification of the Karyadakis procedure. The incision is quite different with thickness of the excised tissue and that of the flap being only $2-3 \mathrm{~mm}^{[30]}$. Though the recurrence rate with this procedure is low, the incidence of wound complications is high.

\section{Limberg flap}

This is a commonly performed off- midline procedure ${ }^{[31]}$. The procedure involves rhomboid excision of the sinus tract along with adjacent tissues followed by developing a rhomboid flap to cover the defect. The flap causes flattening of the natal cleft ensuring lateralisation of the wound. Few surgeons have modified the technique so as to keep the lower end of the flap outside the natal cleft so that the resultant scar lies outside the natal cleft. The recurrence rate with this procedure is very low. However wound dehiscence rates may be high in a few cases depending upon the quality of flap raised.

A comparison of results of median excisional technique versus off midline excision technique reveals promising results with the latter. Therefore off midline excisional techniques are strongly advocated. They are even preferred over the so called minimally invasive excision techniques. Despite the availability of a variety of techniques, it is very difficult for a surgeon to select the so called best technique. No single technique can be considered as the gold standard for cure of pilonidal sinus. Personal experiences with individual techniques and results obtained will continue to be the main guiding force for selecting the surgical technique for treating pilonidal sinus.

Postoperative care is a significant factor which has been ignored by most surgeons thereby leading to recurrence. The maturation of scar takes up to one year from the operation. Until then removal of hair from the back and gluteal region is essential. Electrolysis for hair removal is a better and longstanding option to prevent the hair issue. The area should be washed with soap water twice a day to ensure that accumulated sweat, other cutaneous secretions and loose hair are removed from the natal cleft.

\section{Conclusion}

Pilonidal sinus is disease with high recurrence. Understanding the pathophysiology is pivotal for evolving the best technique. Controlling infection, delineation of the sinus tract and off midline excisional techniques help in preventing wound dehiscence and reducing the recurrence rates.

\section{Conflict of interest: None}

\section{Funding: Nil}

\section{Acknowledgement}

I would like to thank Parth Vagholkar for his help in typesetting the manuscript.

\section{References}

1. Steele SR, Perry WB, Mills S, Buie WD. Practice 
parameters for the management of pilonidal disease. Dis Colon Rectum. 2013; 56:1021-7.

2. Segre D, Pozzo M, Perinotti R, Roche B. Italian Society of Colorectal Surgery: The treatment of pilonidal disease: guidelines of the Italian Society of Colorectal Surgery (SICCR). Tech Coloproctol. 2015; 19:607-13.

3. Al-Khamis A, McCallum I, King PM, Bruce J: Healing by primary versus secondary intention after surgical treatment for pilonidal sinus. Cochrane Database Syst Rev, 2011, CD006213.

4. Enriquez-Navascues JM, Emparanza JI, Alkorta M, Placer $\mathrm{C}$ : Metaanalysis of randomized controlled trials comparing different techniques with primary closure for chronic pilonidal sinus. Tech Coloproctol. 2014; 18:863-72.

5. Plewig G, Kligman AM. In: Acne. Morphogenesis and treatment. Berlin: Springer-Verlag, 1975, 192-3.

6. Bascom J. Pilonidal disease: origin from follicles of hairs and results of follicle removal as treatment. Surgery. 1980; 87:567-72.

7. Karydakis GE. Easy and successful treatment of pilonidal sinus after explanation of its causative process. Aust N Z J Surg. 1992; 62:385-9.

8. Bosche F, Luedi MM, van der Zypen D, Moersdorf P, Krapohl B, Doll D. The hair in the sinus: sharp-ended rootless head hair fragments can be found in large amounts in pilonidal sinus nests. World J Surg. 2018; 42:567-73.

9. Brearley R, Pilonidal Sinus. A new theory of origin. Br J Surg. 1955; 43:62-8. 11. Féré: Bull des la Soc Anat de Paris. 4 Serie. 1878; 3:309.

10. Patey DH, Scarff RW. Pathology of postanal pilonidal sinus; its bearing on treatment. Lancet. 1946; 2:484-6.

11. Guner A, Boz A, Ozkan OF, Ileli O, Kece C, Reis E. Limberg flap versus Bascom cleft lift techniques for sacrococcygeal pilonidal sinus: prospective, randomized trial.World J Surg. 2013; 37:2074-80.

12. Doll D, Matevossian E, Hoenemann C, Hoffmann S. Incision and drainage preceding definite surgery achieves lower 20-year long-term recurrence rate in 583 primary pilonidal sinus surgery patients. J Dtsch Dermatol Ges. 2013; 11:60-4.

13. Jensen SL, Harling H. Prognosis after simple incision and drainage for a first-episode acute pilonidal abscess. Br J Surg. 1988; 75:60-1.

14. Lasithiotakis K, Aghahoseini A, Volanaki D, Peter M, Alexander D: Aspiration for acute pilonidal abscess-a cohort study. J Surg Res. 2018; 223:123-7.

15. Lord PH, Millar DM. Pilonidal Sinus: A Simple Treatment. Br J Surg. 1965; 52:298-300.

16. Gips M, Melki Y, Salem L, Weil R, Sulkes J. Minimal surgery for pilonidal disease using trephines: description of a new technique and long-term outcomes in 1,358 patients. Dis Colon Rectum. 2008; 51:1656-62.

17. Senapati A, Cripps NP, Thompson MR: Bascom's operation in the day-surgical management of symptomatic pilonidal sinus. Br J Surg. 2000; 87:1067-70.

18. Colov EP, Bertelsen CA. Short convalescence and minimal pain after out-patient Bascom's pit-pick operation. Dan Med Bull. 2011; 58:A4348.

19. Iesalnieks I, Deimel S, Schlitt HJ. "Pit picking" surgery for patients with pilonidal disease: mid-term results and risk factors. Chirurg. 2015; 86:482-5.

20. Soll C, Dindo D, Steinemann D, Hauffe T, Clavien PA, Hahnloser D. Sinusectomy for primary pilonidal sinus: less is more. Surgery. 2012; 150:996-1001.
21. Dessily M, Charara F, Ralea S, Allé JL. Pilonidal sinus destruction with a radial laser probe: technique and first Belgian experience. Acta Chir Belg. 2017; 117:164-8.

22. Milone M, Fernandez LM, Musella M, Milone F. Safety and efficacy of minimally invasive video-assisted ablation of pilonidal sinus: a randomized clinical trial. JAMA Surg. 2016; 151:547-53.

23. Dogru O, Camci C, Aygen E, Girgin M, Topuz O: Pilonidal sinus treated with crystallized phenol: an eight-year experience. Dis Colon Rectum. 2004; 47:1934-8.

24. Iesalnieks I, Ommer A, Petersen S, Doll D, Herold A. German national guideline on the management of pilonidal disease. Langenbecks Arch Surg. 2016; 401:599-609.

25. Kelati A, Lagrange S, Le Duff F et al. Laser hair removal after surgery versus surgery alone for the treatment of pilonidal cysts: a retrospective case-control study. J Eur Acad Dermatol Venereol. 2018; 32:2031-3

26. Iesalnieks I, Deimel S, Schlitt HJ. Karydakis flap for recurrent pilonidal disease. World J Surg. 2013; 37:111520.

27. McCallum I, King PM, Bruce J. Healing by primary versus secondary intention after surgical treatment for pilonidal sinus. Cochrane Database Syst Rev, 2007, CD006213.

28. Karydakis GE. New approach to the problem of pilonidal sinus. Lancet. 1973; 2:1414-5.

29. Kitchen PR. Pilonidal sinus: experience with the Karydakis flap. Br J Surg. 1996; 83:1452-5.

30. Bascom J, Bascom T. Utility of the cleft lift procedure in refractory pilonidal disease. Am J Surg 2007; 193:606-9.

31. Cihan A, Ucan BH, Comert M, Cesur A, Cakmak GK, Tascilar O: Superiority of asymmetric modified Limberg flap for surgical treatment of pilonidal disease. Dis Colon Rectum. 2006; 49:244-9. 